\title{
NOTAS METODOLÓGICAS SOBRE EL TRABAJO DE CAMPO ETNOGRÁFICO EN CONTEXTOS DE VIOLENCIA
}

\author{
Adriana Terven Salinas \\ Doctora en Antropología por el CIESAS-DF, \\ licenciada en Etnohistoria/Escuela Nacional de Antropología e Historia, \\ profesora-investigadora en la Facultad de Filosofía de la UAQ \\ Integrante del Foro Latinoamericano de Antropología del Derecho \\ México
}

Karla Rivera Téllez Egresada de la Universidad Autónoma de Querétaro Actualmente es docente de la misma institución y forma parte del área de comunicación del Foro Latinoamericano de Antropología del Derecho Sección México

México

\section{Resumen}

El artículo presenta una revisión y reflexión sobre uno de los pilares fundamentales del quehacer antropológico: el trabajo de campo, particularmente cuando éste se realiza en contextos donde la observación participante, considerada como el paradigma metodológico en la investigación antropológica, se vuelve imposible de llevar a cabo dadas las circunstancias del lugar donde el miedo, la sospecha o el silencio son un componente clave entre la vida y la muerte de los habitantes. El propósito es mostrar la manera en cómo el actual contexto mexicano afecta el desarrollo de investigaciones, la vida de científicos sociales y de las personas involucradas en los estudios. A partir de diversas experiencias de trabajo de campo, se presenta una serie de principios y sugerencias para la realización de trabajo de campo en contextos de violencia, además de criterios para la discusión del tema.

Palabras claves: Contexto, metodología, trabajo de campo, violencia. 


\section{INTRODUCCIÓN}

En la actualidad, la etnografía mexicana y los estudios a profundidad entre las comunidades, como en otros contextos sociales de México y el mundo, se desenvuelven en situaciones y dinámicas extremas de violencia e inseguridad sin precedente (Claudia Jean Harriss Clare, 2015).

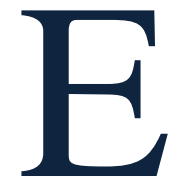

n el

presente texto reflexionamos sobre la realización de trabajo de campo etnográfico en México, tomando como punto de partida el contexto que actualmente define la vida de vastas poblaciones en el país, caracterizado por el miedo, la desconfianza y la inseguridad que ha generado la presencia de las violencias criminal y social en el país. Consideramos que hace falta una reflexión seria y sistematizada que articule tres elementos: la metodología, la posición que ocupa el científico social y el contexto donde se realiza la investigación, con el propósito de proponer mecanismos de seguridad que permitan la continuación de estudios que son necesarios para comprender la dimensión y los grandes retos que enfrenta la sociedad actualmente.

Los contextos de violencias no sólo afectan a las investigaciones que abordan este tema de manera particular, también inciden en la realización de cualquier otro estudio que se ubique en una zona de conflicto (cada vez más extendidas) ya sea rural o urbana, como ha sido el caso de algunos de los textos que se presentan en este dossier, cuyos investigadores enfrentaron situaciones de riesgo.

El artículo se compone de tres apartados, en el primero presentamos los principales acontecimiento que han influido en el actual contexto de violencia en México y señalamos casos de antropólogos que han sido asesinados durante o como parte de su quehacer; en un segundo momento exponemos los resultados de las entrevistas realizadas a distintos investigadores, quienes compartieron sus experiencias de trabajo de campo en contextos que pusieron en riesgo su vida; como un aporte inicial al tema, en la tercera parte presentamos una serie de principios que pueden servir como guía para la realización de trabajo de campo 
en los espacios fuertemente marcados por la presencia de las violencias. ${ }^{1}$

\section{CONTEXTO DE VIOLENCIA EN MÉXICO}

El contexto actual de violencia en México, es el eje sobre el cual gira este artículo, se caracteriza por una "compleja trama de indiferenciaciones miméticas entre grupos de la droga e instituciones estatales" (Pereyra, 2012: 430). De acuerdo con Pereyra, el actual despliegue de violencia sucede en el marco de la transición democrática en México y de las políticas de descentralización a inicios de la década del 2000. Bajo este contexto observamos la imposición de grupos de la droga en las instituciones políticas y de seguridad, los cuales financian las campañas electorales de diversos partidos políticos, que aspiran a cargos estatales y municipales, así como a los gobiernos locales, donde la asignación de recursos no ha sido adecuada para las

\footnotetext{
${ }^{1} \mathrm{Si}$ bien este artículo se centra en experiencias de trabajo de campo en México, la investigación más amplia también documenta casos de trabajo de campo en otras partes del mundo como en África, Asia, Medio Oriente, Norte América, Centro y Sudamérica. El registro de casos abarca desde la década de 1970 y es a partir de aquí que se ha elaborado una guía metodológica para la realización de trabajo de campo en contextos de violencia.

${ }^{2}$ La presencia de los cárteles de droga no es el único factor que detona violencia e inseguridad. Otros factores que inciden en la crisis de violencia que atraviesa el país, según la Comisión Interamericana de Derechos Humanos, es la desigualdad social y económica. En México más del 46\% de la población
}

funciones y competencias que la descentralización les transfirió.

El gobierno ha ubicado al narcotráfico como un problema de seguridad, sin considerar la precariedad de las condiciones socioeconómicas y de servicios de las regiones ${ }^{2}$, concentrándose entonces, en la militarización de la seguridad, lo cual conlleva al control de territorios y poblaciones intensificándose la violencia que ejercen los grupos criminales y el Estado (Pereyra 2012).Desde esta perspectiva, la primera acción que se realizó fue el Operativo Conjunto Michoacán ${ }^{3}$, en el que se enviaron cantidades no tan grandes de soldados -a quienes se les otorgó un aumento salarial del $100 \%$ - para combatir el narcotráfico en aquel estado, dichas acciones tuvieron una enorme difusión televisiva, de radio y medios impresos en 2006.

Sin embargo, la pieza angular y el detonante de la ola de violencia desatada en el país fue la Iniciativa Mérida, un acuerdo

se encuentra en situación de pobreza y pobreza extrema, condición que favorece considerablemente a los indicadores de violencia en el país, pues los bajos salarios, desempleo y las condiciones precarias de vivienda hace que quienes lo padecen -entre ellos migrantes, mujeres, jóvenes, niños y niñas, pueblos indígenas y desplazados- sean más fácilmente captados y explotados por el crimen organizado en gran parte del país.

${ }^{3}$ El Operativo Conjunto Michoacán se llevó a cabo en el estado de Michoacán (oeste del país) debido a que la sociedad civil fue sometida por el crimen organizado y las autoridades locales fueron incapaces de enfrentarse a los grupos criminales que se impusieron haciendo uso de la violencia. 
internacional de seguridad que fue establecido por el ex presidente de los Estados Unidos George Bush y aceptado y firmado en 2008 por México y países de Centroamérica, en la ciudad de Mérida, capital del estado de Yucatán, con la intención de combatir el crimen organizado en los países miembros (Pérez, 2012).Fue así como el ex presidente de México, Felipe Calderón, durante su primer año como mandatario en 2007, lanzó una fuerte ofensiva contra las bandas criminales declarando la Guerra contra el Narcotráfico y autorizando el uso masivo de la fuerza militar (Valenzuela, 2012).

Desde entonces la población mexicana ha sido víctima y testigo de persecuciones, asesinatos, torturas, violaciones, tratos crueles, ejecuciones, mutilaciones, extorsiones, detenciones arbitrarias, desapariciones, secuestros $\mathrm{y}$ migraciones forzadas 5 . De acuerdo a las normas internacionales, los conflictos armados se caracterizan porque el número de bajas rebasa las mil muertes en combate al año. México tiene, e incluso rebasa, las

\footnotetext{
${ }^{4}$ En marzo del 2007 Felipe Calderón pidió la cooperación del gobierno de los Estados Unidos para la lucha contra el narcotráfico, obteniendo de éste financiamiento para cubrir gastos en equipo y armamento como helicópteros, aeronaves de vigilancia y tecnologías de comunicación (Wolf 2011).

${ }^{5}$ El problema de las desapariciones ocupa un lugar importante dentro de las graves consecuencias del conflicto armado. Al respecto, el diario de investigación periodística en versión electrónica, Reporte Índigo, señaló que durante el periodo 2006-
}

características necesarias para declararse en condición de conflicto armado a gran escala, esta situación ha posicionado al país dentro de los tres países con más muertos a causa de conflictos armados internos en el mundo ${ }^{6}$.

Pérez (2012) apunta que en un $60 \%$ de los municipios del país hay gente empleada en el negocio de las drogas, más de medio millón de los habitantes del país están coordinados por los líderes de los cárteles. En la mayoría de los estados del norte la tasa de homicidios ha incrementado notablemente y la aparición de narco-fosas se volvió cada vez más común.Los asesinatos en el estado de Sinaloa aumentaron $543 \%$ y $364 \%$ en el de Chihuahua (De la O, 2012). Ciudad Juárez, por su parte, llegó a alcanzar una tasa de 6,400 homicidios en 2010, lo que le llevó a ganarse el nombre de La capital mundial de los asesinatos. En los estados de Tamaulipas y Durango fueron encontrados 180 y 200 cuerpos mutilados

2012 se tuvo registro de 26,121 personas desaparecidas, cifra que tiempo después fue desmentida y modificada en dos ocasiones por el gobierno federal. Este indicó, primeramente, que la cifra oficial era de 18,955 desaparecidos, número que meses después fue nuevamente alterado por el mismo, indicando finalmente que el resultado oficial de desaparecidos durante aquel sexenio fue de 23,271.

6 Consultado en Mayo del 2016 en la página electrónica del Instituto Internacional de Estudios Estratégicos de Londres. 
respectivamente dentro de narco-fosas clandestinas (Wolf, 2011) ${ }^{7}$.

El regreso del Partido Revolucionario Institucional (PRI) con la entrada de Enrique Peña Nieto a la presidencia en diciembre de 2012, no trajo cambios sustantivos en la estrategia de seguridad pública. Se presume que el modelo implementado por Calderón es el mismo que ha puesto en marcha el actual presidente de la república ${ }^{8}$, teniendo como resultado un incremento incluso más alarmante en las tasas de desapariciones, persecuciones, torturas, maltratos, detenciones arbitrarias y asesinatos en todo el territorio del país 9 .

El incremento de la violencia en México por la constante disputa de poder sobre el territorio ha generado varios tipos de violencia, De la O y Medina (2012) identifican tres categorías: 1) la generada por la lucha de cártel contra cártel, 2) cártel contra gobierno y 3) cártel contra población civil. Dentro de este rubro, cabe mencionar que las fuerzas de seguridad, en su afán por combatir a los narcotraficantes, las agresiones causadas por las fuerzas

\footnotetext{
7 Cabe señalar que, según la Global Burden of Armed Violence 2015, de los 25 países con mayor tasa de feminicidios, 10 se encuentran en América Latina; México entre los primero cinco con una muerte violenta en promedio cada cuatro horas.

${ }^{8}$ Movimiento por la Paz con Justicia y Dignidad (MPJD).

9 De acuerdo a Instituto Nacional de Estadística, Geografía e Informática, durante el lapso
}

militares y policiacas hacia civiles han violado severamente los derechos humanos de la población abusando del poder que les confiere la ley y llevando a cabo detenciones arbitrarias, registros domiciliarios ilegales, golpizas, violaciones, secuestros, torturas, desapariciones forzadas y asesinatos ilícitos hechos al azar haciendo estos escenarios cada vez más comunes entre la población.

La grave secuela del clima de terror e impunidad que reina en el país ha abierto el camino a la banalización y normalización gradual de la violencia (Guilles, 2015). Este clima de hostilidad altera significativamente la organización social, familiar y económica: el crimen organizado irrumpe en localidades enteras, incide en el uso de la tierra, se apropia de espacios públicos y viola los derechos humanos redefiniendo la vida cotidiana de miles de personas todos los días (Valenzuela, 2012).

Ante esta situación, la pertinencia de los estudios antropológicos y sociales en relación al fenómeno de la violencia, o sobre temas que se ubican en contextos de violencia, se vuelve crucial, ya que para el

comprendido de 1990 a 1999 hubo en promedio 14, 767 homicidios. Luego de este periodo hubo un deceso, del 2000 al 2007 hubo en promedio 11,395 homicidios y fue a partir del 2008 que hubo un incremento considerable en las cifras, teniendo como pico el año 2011, que terminó con 27,213 homicidios. En total, durante el período abarcado del 2008 al 2015, según datos oficiales, hubo en promedio 21,003 homicidios. 
análisis y comprensión de las problemáticas, tendrán que considerarse las consecuencias de carácter social y cultural en los individuos en la generación de problemas de identidad, pertenencia social, inestabilidad de rol o desequilibrio en las relaciones familiares que se presentan en estos lugares. Surgen entonces dificultades metodológicas a la hora de realizar trabajo de campo en sitios donde el miedo, la sospecha o el silencio son componentes claves entre la vida y la muerte. Ser antropólogo se vuelve cada vez más una tarea de alto riesgo, ejemplo de ello son los casos de homicidios de antropólogos que comenzaron a registrarse desde el 2008, periodo que coincide con el inicio del pico más alto de homicidios en el país según el Instituto Nacional de Estadística y Geografía e Informática (INEGI).

El primer caso registrado hacia un antropólogo en el actual contexto de violencia fue el de Miguel Ángel Gutiérrez Ávila, investigador de la Universidad Autónoma de Guerrero y activista en pro de los derechos indígenas, quien fue golpeado hasta la muerte en una carretera del estado de Guerrero mientras regresaba de hacer trabajo de campo. Su muerte estuvo relacionada con la filmación y

${ }^{10}$ Agricultura Nacional de Veracruz, S. A., empresa dedicada a la fabricación de fertilizantes y pesticidas. En 1991 hubo un corto circuito en la fábrica, mismo que devino en un incendio descontrolado $\mathrm{y}$ documentación que hizo sobre los ataques y agresiones por parte de la Agencia Federal de Investigación (AFI) hacia la radio indígena comunitaria "La voz del agua" en la que se denunciaban inconformidades por parte de la comunidad y con la cual el investigador colaboró desde su fundación.

$$
\text { El segundo caso se suscitó el mismo }
$$
año con Eric Cipriano, alumno de la Escuela Nacional de Antropología e Historia (ENAH), quien fue asesinado mientras realizaba trabajo de campo en Maruata, Michoacán, una de las entidades con mayor índice de inseguridad y violencia derivada de la guerra contra el narcotráfico. Un año después, en 2009, desapareció Luis Cisneros, estudiante de la misma institución quien tenía una vida política activamilitante y cuyo caso no ha sido resuelto ni su cuerpo encontrado desde entonces.

En 2012, José Luis Blanco, antropólogo catedrático de la Universidad Veracruzana, fue asesinado en su casa. El académico realizó trabajos de investigación en zonas rurales marginales en el estado de Veracruz en torno a la conservación del patrimonio histórico-cultural y medio ambiente y encabezó un importante estudio en torno a los afectados por la explosión de Anaversa ${ }^{10}$, trabajo que le sirvió para llevar

finalmente en una explosión que originó el derrame y combustión de miles de litros de químicos altamente tóxicos causando la muerte de miles de 
el caso a la Corte Interamericana de Derechos Humanos. Las causas de su muerte siguen levantando sospechas y se cree que el entonces gobernador del mismo estado, Javier Duarte ${ }^{11}$, fue quien dio la orden de asesinato.

En último caso de crimen del que se tiene registro tiene que ver con el multihomicidio de la colonia Narvarteen la Ciudad de México en 2015 en el que, luego de varias amenazas y hostigamiento por parte del gobierno del estado de Veracruz, la antropóloga y activista Nadia Vera junto con tres personas más fueron asesinadas ${ }^{12}$. El asesinato estuvo vinculado a las múltiples denuncias que ella realizaba constantemente en torno a la carencia de derechos humanos en la misma entidad federativa responsabilizando a Javier Duarte, del deterioro de los sistemas de seguridad en la región. En un video circulado en la red, la activista responsabiliza al mismo mandatario de cualquier agravio que ella y sus compañeros pudieran sufrir.

Frente a estos casos, se presenta como urgente reflexionar sobre la importancia de la investigación

personas y malformaciones genéticas en las generaciones sucesoras.

11 Ex gobernador del estado de Veracruz (20102016) actualmente enfrenta un proceso penal por vínculos con el crimen organizado, abuso de autoridad y estar relacionado con el homicidio de algunos periodistas en la misma entidad federativa. antropológica articulada con la necesidad de plantear metodologías que permitan abordar estos contextos. Feixa y Ferrándiz (2004), y Valenzuela (2012) coinciden en la trascendencia de hacer uso de las herramientas que nos concede la disciplina para describir, analizar y comprender las tramas de la violencia para denunciarlas y contribuir a generar nuevos marcos de convivencia, nuevos proyectos de nación que sean más incluyentes y equitativos. Partiendo de esto, consideramos que es necesario asumir la investigación académica como un compromiso político, encontrar en el trabajo de campo un lugar de militancia y compromiso ético desde donde se pueda escribir contra el terror.

\section{TRABAJO DE CAMPO EN CONTEXTOS DE VIOLENCIA}

Para iniciar una reflexión metodológica sobre la realización de trabajo de campo en contextos de violencia, se consultaron publicaciones sobre investigaciones a nivel internacional que se realizaron en lugares que ubicamos en

\footnotetext{
12 Otra de las personas asesinadas junto con Nadia Vera fue el foto-periodista Rubén Espinosa, quien también fue víctima de abusos y hostigamientos por parte de las autoridades por publicar fotos incómodas para el gobierno de Javier Duarte. Es necesario señalar en este punto que México se ha convertido durante los últimos años en uno de los tres países más peligrosos para ejercer el periodismo.
} 
dichos contextos y sobre temas relacionados con el fenómeno de la violencia $^{13}$. En el caso de las experiencias de trabajo de campo en México, la mayoría de las publicaciones no presentaban una sistematización metodológica sobre los retos enfrentados durante las investigaciones, se decidió contactar con antropólogos mexicanos, quienes fueron seleccionados a partir de sus temas de estudio o por las regiones donde trabajan, accedieron a participar ocho mujeres y tres hombres adscritos al Centro de Investigaciones y Estudios Superiores en Antropología Social (CIESAS), Colegio de San Luis, Universidad de Guadalajara, Universidad Autónoma Metropolitana y el Colegio de Michoacán; y una activista estudiante de la licenciatura en Antropología de la Universidad Autónoma de Querétaro ${ }^{14}$.

De acuerdo con la información facilitada, los temas de estudio en los que el contexto de violencia y sus consecuencias sociales estuvieron presentes como

13 Durín 2012; De la O 2012; Feldman 1995; Ferrándiz 2008; Gavilán, 2012; Gaskew, 2009;Gill, 2004; Gilles 2015; Goldstein 2014; Green 1995; Howell 2012; Jimeno 2011; Kovats-Bernat 2002; Lee-Treweek y Linkogle 2000; Manz 1995; Martínez 2015; Nateras ,2010; Nash, 2012; Nordstrom 1995; Nordstrom y Robben, 1995; Olujic, 1995; Omidian, 2009, Pieke 1995; Rodgers, 1997; Sandoval, 2012; Swedwnburg, 1995; Zavala, 2014.

${ }^{14}$ La dinámica de trabajo con las y los participantes consistió en la realización de un cuestionario en línea a 12 personas, las preguntas indagaban sobre los tema de investigación, lugar dónde se realizó, limitantes y modeladores en el ejercicio de hacer trabajo de campo, se relacionan con temas de ciudadanía, contrabando, migración, sistemas agrícolas, desplazamiento forzado, procesos judiciales, desapariciones forzadas, sociedad civil organizada, derechos humanos, vida callejera y refugiados políticos $^{15}$.

El primer caso que presentamos es el de una investigación sobre contrabando y comercio transfronterizo que se realizó en la frontera norte del país por un investigador del CIESAS, el cual tuvo como objetivo analizar el flujo de mercancía transfronterizo entre Nuevo León, Tamaulipas y Texas, zona donde persiste una fuerte presencia de narcotraficantes. Durante el trabajo de campo el investigador se enfrentó a un contexto donde el común denominador eran los enfrentamientos entre cárteles, extorciones, secuestros, impunidad y violencia armada, pues los grupos

financiamiento, condiciones de entrada y establecimiento en el lugar donde se realizó el trabajo de campo, formas de entablar relaciones (previamente y en el lugar), formas de abordaje y registro de la información, conocimiento (previo e in situ) del lugar y sus implicaciones en el trabajo de campo, situaciones de violencia presenciadas o vividas, cómo se actuó frente a esto. Posteriormente accedieron a entrevista siete personas.

${ }^{15}$ Respecto de las personas que participaron, algunos de ellos solicitaron anonimato, en los casos donde aparece el nombre con apellido refiere a los nombres reales, cuando solo aparece el nombre se trata de un pseudónimo. 
criminales se habían infiltrado en la organización del comercio de "fayuca"16.

"Debido al incremento de la violencia en la carretera entre Reynosa y Monterrey en Marzo del 2010 me vi obligado a suspender las actividades de trabajo de campo que implicaban trasladarme semanalmente entre ambas ciudades para cruzar la frontera de Texas con México. La decisión fue dramática. Sentí impotencia no sólo como antropólogo sino como ciudadano y habitante de este país. Tomé la decisión de no viajar más mientras me encontraba en Texas cuando me enteré de los enfrentamientos ocurridos en la carretera la noche anterior a mi regreso a Monterrey. Las horas previas a mi retorno fueron intensas" (Efrén Sandoval. Comunicación personal, 22 de septiembre de 2016).

Como consecuencia del estallido de la guerra en las calles de las ciudades, poblaciones enteras fueron empujadas a trasladarse a otros lugares debido a la inseguridad que vivían cotidianamente siendo testigos oculares de crímenes, víctimas de amenazas físicas, coacción directa, degradación de la calidad de vida o falta de oportunidades laborales. Las

\footnotetext{
${ }^{16}$ En México la palabra "fayuca" refiere a productos que se compran en Estados Unidos y son introducidos al país por contrabandistas con fines comerciales.
}

consecuencias sociales de los agravios son diversas y han sido abordados desde diferentes aristas por antropólogos y científicos sociales ${ }^{17}$, que fueron víctimas $y$ testigos de hostigamientos y asaltos a mano armada mientras realizaban trabajo de campo bajo la presencia de militares y marinos que trataban de "arreglar" la situación frente a los cárteles de droga causando un éxodo masivo, principalmente en la zona norte del país.

Sobre el tema, Camila, una antropóloga dedicada al estudio del fenómeno migratorio en el noreste mexicano que se dio a partir de la guerra contra el narcotráfico, comenta que la población había vivido desde entonces un crecimiento exponencial de la violencia en la vida cotidiana, en la cual diariamente había enfrentamientos entre grupos rivales o con el gobierno. Lo anterior produjo que sólo en cuatro años hubiera cerca de $40 \mathrm{mil}$ mexicanos pidiendo asilo político en Estados Unidos y Canadá principalmente.

Derivado de tal trabajo de
investigación,
internacional le pidió la redacción de un texto para la publicación de un libro acerca del tema del desplazamiento interno

17 Pieke, 1995; Nordstrom, 1995; Green, 1995; Winkler y Hanke, 1995; Olujic, 1995; Durin, 2012; De la O y Medina, 2012; Gaskew, 2009; Jimeno, 2011; Nateras, 2010; Nash, 2012; Omidian, 2009; Rodgers, 1997; Sandoval, 2012; Zavala, 2014. 
forzado en México, asunto en el que se tocaría el tema de la narcoviolencia. En sus escritos presentó el resultado cuantitativo de los desplazados en México en ciertos estados del norte. A raíz de esta investigación fue contactada por un reconocido periodista para ser entrevistada sobre su trabajo de investigación. Luego de una serie de charlas, el material se publicó un mes después de la entrada de Enrique Peña Nieto a la presidencia, en un periódico de circulación nacional durante tres días. Al segundo día de la publicación, la investigadora recibió una carta de la misma organización para la cual realizó el texto sobre desplazados (la nueva presidenta de la organización había entrado con el cambio de gobierno), en la que se le informaba que la publicación de su libro sería censurada ${ }^{18}$ :

"Para mí fue muy claro el mensaje, fue muy claro que hubo comunicación entre el Ejecutivo y las oficinas de la organización [...] Fue como un balde de agua fría en la espalda. Durante el 2012 hubo un spot publicitario de presidencia que decía "México está en paz". Había que tapar esas situaciones $y$ por eso no eran bienvenidos los desplazados. Entonces entendí claramente que era un tema del que no se quería hablar. Sentí miedo. En

18 El libro fue censurado de inmediato por la inconveniente información que resguardaba, sin embargo el movimiento de relaciones entre la institución que respaldaba a la antropóloga y un situaciones así, debes esperar un ratito para que se olviden de ti, luego puedes retomar" (Camila. Comunicación personal, 16 de Septiembre de 2016).

Otro de los investigadores entrevistados ha realizado trabajo de campo en las reservas de hidrocarburos y minerales que albergan algunas zonas rurales, situación que ha abierto el camino a diferentes empresas extranjeras para explotar dichos recursos despojando a la población de éstos, lo que ha generado un ambiente tenso, pues el conflicto implica los intereses tanto de los pobladores, narcoempresarios, caciques, políticos $\mathrm{y}$ gobernantes que ven en ellos una fuente de riqueza y se la disputan, muchas veces, a muerte.

"Esto ha sido característico de esta región, el uso de la violencia tanto por parte de los caciques como de la misma gente de las comunidades como una respuesta a esta presión que han tenido toda su vida y una violencia de estado donde siempre te encuentras gente de gobernación y militares en las comunidades. Mi trabajo de investigación gira en torno a la posible implementación de proyectos de neoextractivismo [...] me llevaron a visitar pozos petroleros y al regresar a la cabecera

importante político que trabajó la ley de desplazamiento forzado en Chiapas (sur del país) logró que el Senado de la República aprobara la publicación del libro un año después de la censura. 
del pueblo ya había una camioneta con militares esperándonos. No nos dijeron nada pero seguramente tomaron nota de lo que andábamos haciendo" (Eduardo. Comunicación personal, 12 de Agosto de 2016).

Los casos anteriores revelan el "control ilegal de las poblaciones y la aplicación de las políticas de seguridad que ponen en riesgo la vida de las personas" (Pereyra, 2012: 431), en este contexto, el Estado en vez de garantizar la seguridad de los antropólogos (activistas y periodistas), representa un sujeto amenazante junto con el crimen organizado, al censurar la realización de investigaciones por medios violentos.

Otro componente de la desatada crisis de violencia por la que atraviesa el país tiene que ver con feminicidios asesinatos misóginos de mujeres que ocurren en circunstancias específicas y que se perpetran en condiciones donde el Estado $\mathrm{y}$ sus agentes los mantienen impunes- ${ }^{19}$. Según cifras del Instituto Nacional de Estadística Geografía e Informática (INEGI), se estima que durante 2013 y 2014 siete mujeres fueron asesinadas diariamente. La situación y sus consecuencias han sido también estudiadas

\footnotetext{
${ }^{19} \mathrm{http} / / /$ www.inegi.org.mx/saladeprensa/aproposito/ 2015/violencia0.pdf
}

por científicos sociales. Algunas de estas investigaciones han estado acompañadas de amenazas por parte de quienes detentan el poder, a quienes no les conviene que sus administraciones sean catalogadas como violentas $\mathrm{o}$ incompetentes en cuanto a seguridad pública.

Un ejemplo claro es el de Aleida Quintana, antropóloga dedicada a investigar y dar seguimiento a familiares de víctimas de desapariciones y feminicidios en el estado de Querétaro, ha recibido múltiples amenazas de violación y muerte por parte del gobierno del estado, administración que encuentra poco conveniente que el nombre de la política queretana se manche. La antropóloga narró que fue víctima de persecuciones en auto y a pie, amenazas vía telefónica y agresiones físicas en dos ocasiones por parte de quienes la vigilaban.

"Una vez en un autobús dos tipos me agarraron del cabello y me amenazaron. La otra en la Procuraduría ${ }^{20}$, me patearon. He recibido llamadas con amenazas de muerte, "date por muerta", me decían. La vigilancia sigue a pesar de la seguridad con la que cuento por parte de SEGOB a través de un mecanismo de protección de defensores de derechos humanos y periodistas a través de la ONU. No confío

20 Actualmente Fiscalía General de Justicia, responsable de la seguridad pública y la procuración de justicia. 
en ellos, pero al menos me permiten tener movilidad" (Aleida Quintana. Comunicación personal, 4 de julio de 2016).

Ante esto y después de cuatro años de dar seguimiento a víctimas y familiares de las mismas, señala:

"Cuando trabajas con violencia te encuentras con que tu agresor puede ser casi cualquiera, desde el esposo que le pega a la esposa, o si trabajas con feminicidios puede que sean las transnacionales, el mismo Estado o el narcotráfico" (Aleida Quintana. Comunicación personal, 4 de julio de 2016).

Respecto de las desapariciones forzadas, tema que ha puesto a México en la mira internacional no sólo por la falta de claridad y concordancia de las cifras oficiales, sino también porque a diferencia de lo que se vivió en el país durante la Guerra Sucia, donde las desapariciones se cometían con motivos políticos, actualmente no sólo se desaparecen líderes sociales, activistas políticos o integrantes de grupos insurgentes, también se extiende a amplios sectores de la población ${ }^{21}$. La problemática no sólo representa un riesgo para las víctimas directas, sino para los familiares que en busca de sus desaparecidos por medio de organizaciones civiles o independientemente, reciben

\footnotetext{
${ }^{21}$ Violaciones Graves a Derechos Humanos en la Guerra contra las Drogas. Comisión Mexicana de Defensa y Promoción de Derechos Humanos.
}

constantes amenazas de muerte por parte de entidades gubernamentales.

Siendo esto último no exclusivo de los familiares de las víctimas, existen quienes han llevado a cabo investigaciones sobre sociedad civil organizada en estos contextos. Gabriela, antropóloga e investigadora del tema en diversas ciudades del país, señala:

$$
\text { "[...] cuando te empiezas a }
$$
introducir al tema te das cuenta de que son temas bastante peligrosos o que están ligados al crimen organizado o con el mismo Estado, entonces uno tiene que ir pensando en cómo ir replanteando el tema y toda la investigación para que pueda llevarse a cabo sin poner la vida en riesgo. Cuando escribía el epílogo de mi tesis, pasó el multi-homicidio de la colonia Narvarte muy cerca de mi casa. Yo conocía a unos de ellos, el hecho de cómo fueron violentados me hizo repensar mi investigación" (Gabriela. Comunicación personal, 22 de Agosto, 2016).

A partir de los casos anteriormente expuestos, se evidencia el riesgo que corren quienes pretenden hacer etnografía -o algún tipo de incidencia en su práctica profesional como antropólogos/as- en tiempos violentos y ambientes hostiles, advirtiéndose en la 
falta de medidas de seguridad para la realización de dichas investigaciones.

Consideramos que desde la reflexión metodológica se pueden plantear mecanismos para la realización de trabajo de campo en estos contextos, preguntándonos ¿qué estrategias de investigación debemos seleccionar frente a tales "accidentes antropológicos"? ${ }^{22}$ Frente a dichos sucesos algunos investigadores han optado por seguir con el plan inicial, como si nada hubiera cambiado, otros prefieren ir a lugares más seguros, otros abandonan el campo y regresan a casa, aún así hay quienes deciden permanecer en el área de estudio (Nordstrom y Robben, 1995), tratando de ajustar su plan metodológico para hacer del peligro su nuevo marco contextual.

Como se ha mostrado, el contexto actual del país representa un desafío para el trabajo de investigación, pues los obstáculos derivados de las políticas de seguridad adoptadas por los últimos presidentes han redefinido las condiciones de vida en comunidades enteras y por tanto las formas de hacer etnografía. En este sentido, comentan De la O y Medina (2012) que la antropología en México tiene alcances diferentes y se hace necesario

22 Frank Sieke (1995) define los accidentes antropológicos como un conjunto eventos inesperados durante trabajo de campo. repensar y reaprender a hacer investigación en realidades sociales de este carácter, obligándonos a evaluar y moldear nuestros marcos teóricos y metodológicos clásicos, ante la imposibilidad de tener un acercamiento a determinadas comunidades en situaciones de conflicto, debido a la desconfianza y riesgo que existe bajo estos contextos. Al respecto, Kovats-Bernat (2002) apuntan que "lo que se necesita es una estrategia pragmática para lidiar con la amenaza de seguridad y el bienestar de los antropólogos e informantes que trabajan en medio de la amenaza de la violencia" (Kovats-Bernat, 2002: 1); así como la adopción de nuevas técnicas de investigación y estrategias que reten las normas y éticas convencionales de la disciplina para negociar el intercambio de datos bajo circunstancias de peligro.

Es difícil realizar un trabajo de campo en sitios donde el miedo, la sospecha, el secreto y el silencio son componentes esenciales y crónicos de la memoria e interacción social, es por eso que en contextos de peligro, a medida que aumenta la intensidad de la violencia, lo hacen al mismo tiempo las incertidumbres y peligros de llevar a cabo una investigación tanto para el antropólogo como para los informantes. Así, la investigación en 
contextos de violencia abre nuevos paradigmas y nos enfrenta a situaciones en las que debemos extremar precauciones haciendo del trabajo de campo un "campo de minas"- como sugiere Ferrándiz (2008), en el cual debemos de actuar con cautela, incrementar la precisión en nuestro quehacer, ser precavidos y capaces de anticipar los peligros y dificultades que se puedan presentar durante la investigación, considerando una forma más heterogénea y menos rígida que se ajuste a las necesidades $\mathrm{y}$ vicisitudes que vayan surgiendo en el proceso.

Para cerrar este apartado, es crucial subrayar que, dadas las circunstancias de descomposición de las condiciones de vida derivada de la crisis social y global a la que nos enfrentamos en el siglo XXI, se vuelve necesario, como menciona Rosemberg (2012), ser capaces de crear etnografías de indignación, en las cuales los relatos contados e historias de vida sirvan para evocar la memoria de los muertos y desaparecidos y que las etnografías sirvan para hacer un llamado a la justicia y en contra de la represión.

Principios y sugerencias para el trabajo de campo en contextos de violencia.

Es importante para los investigadores entender que durante periodos de crisis exista un grado de desconfianza dentro de las comunidades con las que se trabaja. A continuación presentamos una serie de principios $\mathrm{y}$ sugerencias que pueden ser útiles para quienes realizan trabajo de campo en contextos de violencia, pero también para plantear criterios para la discusión del tema. De inicio, es importante tener siempre credenciales de identificación con información de la institución u organización a la cual pertenecemos y tratar de tener un respaldo de la misma por medio de cartas de presentación que avalen nuestro quehacer como antropólogos.

Zavala (2014), quien trabajó con jóvenes pandilleros de Cancún, decidió acudir a la Secretaría de Seguridad Pública del gobierno estatal con el fin de obtener información sobre las pandillas en la ciudad. Como estrategia de seguridad ante su posible detención al estar vinculada con las pandillas, tuvo que proporcionar información personal, llenar registros, entregar cartas de presentación de la institución, tomar huellas dactilares y hacer una exposición sobre su quehacer antropológico. Esto le permitió credibilidad en el trabajo de investigación, pues había cierta renuencia por parte del gobierno estatal para darle información.

En una época donde impera el miedo generalizado, también es importante generar lazos de confianza, primero por medio de la información que brindemos a la 
comunidad sobre nuestro trabajo y nuestro estatus como investigadores, pero también por medio de la escucha, pues según la experiencia de la antropóloga Jaqueline Garza, en su investigación con familiares de desaparecidos, una vez que aprendemos a escuchar a nuestros sujetos de estudio, ellos se abrirán y se podrá comenzar a generar un sentimiento de confianza mutua que favorezca la investigación.

En este sentido, Zavala (2014) menciona la importancia de compartir actividades cotidianas e informales con los participantes como pueden ser ir a cortarse el cabello, barrer la calle o comer con los vecinos, pues el afianzamiento de las relaciones y la reducción de las brechas entre antropólogo y participantes tiene mucho que ver con la manera en cómo nos acercamos a los sujetos.

En un contexto urbano y trabajando en ambientes callejeros en la ciudad de México, Danielle Strickland optó por elegir a los líderes pandilleros que tuvieran status como informantes clave, esto como estrategia de seguridad para llevar a cabo su investigación sin correr riesgos. En consecuencia, el fortalecimiento de las relaciones que mantenemos con los sujetos es importante, pues en caso de tener que salir de la comunidad de estudio repentinamente a causa del peligro que el contexto presenta, el grupo podría ayudar al investigador a salir, escapar o pasar desapercibido ante cualquier amenaza.

Una vez afianzados los vínculos con los informantes clave es importante, señala Rodgers (1997), relacionarse poco a poco con la población tomando paseos por las calles, identificando gente y entablando diálogo con los mismos tratando de actuar "etnográficamente", es decir, adoptar la cultura local. Observar cómo la población habla y lidia con los demás nos ayudará no sólo a ganar su confianza, sino a identificar las medidas de seguridad que ellos mismos tienen para enfrentar los peligros de la vida diaria, cuáles conversaciones -y silenciosson importantes, qué personas evitar y cómo movernos para eludir ser blanco de sospecha o víctima de algún crimen o delito.

Es importante saber que muchas veces las personas del lugar desarrollan una especie de mapa mental sobre su territorio que les permite reconocer personas, lugares seguros y las horas adecuadas para pasar por ciertas zonas (Goldstein, 2014), por eso, poder identificar áreas de riesgo y "zonas seguras" con la ayuda de los informantes durante trabajo de campo, se vuelve crucial para prevenir agresiones.

Fernanda Arias, antropóloga que trabaja en contextos urbanos aporta una metodología que ella misma empleó en colonias catalogadas como peligrosas en la ciudad de Querétaro. En las colonias, 
señala, hay divisiones internas basadas en rivalidades tanto de grupos de pandillas como de vecinos, así, la concepción de "zona peligrosa" depende casi por completo de la zona en la que se habite o la pandilla a la que se pertenece. La antropóloga cayó en cuenta de lo anterior y la estrategia fue sondear, por un lado, a las personas que vivían en la zona más alta del barrio para saber cuáles eran las zonas consideradas por ellos como "peligrosas".

Este método fue utilizado con las personas que vivían en la zona baja y con integrantes de pandillas, teniendo como resultado tres mapas que derivaron de lo que cada grupo consideraba como "zona de riesgo". Dichos mapas fueron impresos en láminas de acetato marcando con diferentes colores las zonas identificadas por los grupos, así al sobreponerlos conformaban un solo mapa que mostraba el total de las zonas peligrosos mencionadas por todos los sondeados. Con base en esto, la antropóloga y su equipo trabajaron en las zonas que aparecían del todo como "libres de riesgo".

Dentro de otras consideraciones a tomar en cuenta es oportuno mencionar que debemos evitar a toda costa el consumo de drogas y alcohol, pues como ya se ha mencionado, en ambientes donde el miedo es generalizado, cualquier movimiento fuera de lugar puede poner al investigador en un rol de víctima. En este caso, al comprar sustancias ilícitas cabe la posibilidad de que el etnógrafo sea identificado como consumidor frecuente o proveedor de droga.

Haciendo hincapié en la importancia que tiene la inmersión del investigador en el grupo objeto de estudio, la observación participante en cotidianidades agresivas se vuelve difícil, por lo que es importante estimar que si la situación lo amerita, tendremos que limitar nuestra intervención a mirar y escuchar considerando la creatividad y flexibilidad como importantes estrategias de supervivencia. Siguiendo con la misma idea, Kovats-Bernat (2004) menciona que si se va trabajar en áreas peligrosas, se debe comenzar con un cambio desde cómo es definida la metodología, es decir, ya no como un ensamblado rígido, sino como una práctica ecléctica, flexible, diversificadora e integradora, que contemple formas de escape ante el peligro para salir bien librados. Como menciona Durand (2014), el oficio se aprende con la práctica y si no hay imaginación sociológica, difícilmente se pueden encontrar caminos nuevos, soluciones diferentes y enfoques originales.

En este sentido, hay que reconocer que en ocasiones los objetivos que se persiguen inicialmente no siempre podrán alcanzarse debido a la poca información que hay, o a las limitantes que la vida cotidiana 
en contextos de violencia representan. Al respecto Danielle Strickland señala la impotencia sentida al no poder conocer los escenarios en los que se desenvolvían sus sujetos de estudio después de las ocho de la noche en el barrio de Tepito ${ }^{23}$.

El trabajo de campo bajo estos contextos rompe con toda regla occidental, señala Gill (2004). Si tuviéramos que seguir al pie de la letra todas las precauciones como no ir a lugares desconocidos $\sin$ compañía, no confiar en extraños o no estar fuera tan tarde, entonces no se podría avanzar en la investigación, es por eso que también es necesario tomar ciertos riesgos y encontrar un punto medio que nos ayude a discernir entre los límites de los accidentes antropológicos y las oportunidades que se nos presentan.

En zonas de conflicto donde los derechos humanos y las garantías individuales son borrados, es importante recordar que el trabajo de los antropólogos también puede ser usado en contra de la gente con la que trabajamos, por lo tanto debemos brindar protección y seguridad a quienes participan de la investigación, teniendo en cuenta la responsabilidad ética de emprender una evaluación de los riesgos que los participantes y la sociedad en general podrían enfrentar como

\footnotetext{
${ }^{23}$ Barrio ubicado en la Ciudad de México con alta
} actividad comercial y altos índices de delincuencia. consecuencia de la investigación (LeeTreweek y Linkogle, 2000). Es por eso necesario cambiar los nombres y omitir incluso en el diario de campo y notasnombres de lugares, empresas o cualquier información por muy importante que sea, que pueda amenazar la seguridad de los participantes, pues los diarios de campo guardan mucha información que puede ser usada en contra de la población, e incluso de nosotros mismos.

Al respecto, la antropóloga Camila, señala la pertinencia de borrar archivos de audio después de tomar notas si es necesario, pues esto es parte del contrato de confianza que se establece entre el investigador y el informante cuando se tiene que lidiar con información importante que puede ser factor clave entre la vida y la muerte:

"Debemos recordarnos diariamente que algunas de las cosas que anotamos pueden significar acoso, exilio, tortura o muerte para nuestros informantes o para nosotros mismos" (Kovats-Bernat,2004:9).

Respecto al diario de campo y libreta de notas, diversos investigadores coinciden en que éstas son las herramientas básicas del antropólogo, sin embargo hay que saber ser prudente y elegir el momento adecuado para escribir en el diario o tomar 
notas. Por seguridad se recomienda no escribir delante de las personas con quienes trabajamos, sino hacer uso de la retención de memoria o anotar solamente palabras clave que se puedan relacionar fácilmente con el acontecimiento que se desea describir para después.

Siguiendo en este rubro, es recomendable entrenar la destreza para crear jotting notes, es decir, anotaciones de palabras clave, abreviaciones, símbolos, frases cortas e incluso dibujos que además de hacernos recordar la idea que en el momento no pudimos escribir, pueden servir como método de seguridad al ser incompresibles para quienes pretendan leerlas, pues como ha sido ya mencionado, las notas de campo pueden contener información sensible que podría ser empleada en contra del antropólogo o sus informantes (Kovats-Bernat,2004).

Para la escritura de este tipo de anotaciones, algunos etnógrafos prefieren hojas de papel dobladas que puedan ser fácilmente guardados y/o desechadas, o bien libretas pequeñas que quepan en los bolsillos, recientemente hay quienes han optado por hacerlo mediante dispositivos electrónicos como celulares, tablets o grabadoras (Emerson et al, 2011), es importante asegurarnos de que sólo el propietario del aparato tenga acceso a estas herramientas creando contraseñas de acceso seguras.

"Uno se vuelve un poco paranoico. En mi computadora a veces la información se bloqueaba o se cerraba, sobre todo cuando estaba en internet" (Gabriela, comunicación personal, 22 de Agosto del 2016).

Es importante en este punto señalar que en todo momento se debe evitar, incluso en jotting notes, anotar el nombre real de los participantes, para esto se pueden usar pseudónimos, número e incluso claves. Jenkins (en Feldman 1995), en este sentido, sugiere que uno debe portar solamente la información y notas del día.

Al hacer uso de dispositivos electrónicos adquirimos la obligación de respaldar toda la información que se recolecta ya sea en disco duro, usb, tarjetas de memoria, dropbox o correo electrónico, sin embargo se deben extremar precauciones con la información que ahí se deposita, pues como ya se ha mencionado, es fácil que la información se filtre en la red y ésta llegue a manos de quienes puedan ponernos en riesgo a nosotros o a nuestros informantes. Por eso, como opciones alternas y más seguras se puede hacer uso de plataformas específicas de manera confiable como Jitsi para hacer video llamadas de forma segura, pad.riseuo.net para generar documentos compartidos 
privados, Encrypt para enviar mensajes de texto confidencialmente o Telegram como sustituto de Whatsapp. A pesar de todas estas recomendaciones de programas $y$ plataformas específicas, la antropóloga y activista Aleida Quintana señala que es importante tener siempre crédito en el celular y si es necesario contar con dos dispositivos en diferentes compañías telefónicas por si alguna falla, no contestar números desconocidos y siempre tener un directorio en el bolso o mochila por si existe algún tipo de agresión física se pueda contactar rápidamente a algún familiar o amigo.

Sobre el uso de la cámara fotográfica o de video, en contextos de violencia es preferible no emplearla y en caso de ser necesario sería más adecuado utilizar un dispositivo móvil con cámara, pues es más pequeño, manipulable y fácil de esconder. Camila, quien trabajó con familias de desplazados, recomienda únicamente tomar fotos de recorridos $\mathrm{y}$ lugares, pero nunca de las personas, pues esto, además de incomodar, podría exponer la vida de nuestros informantes en ciertos contextos.

En contextos donde se trabaja con emociones fuertes, es necesario que el investigador sea sensible con las personas que le comparten experiencias de violencias y sobre cómo puede ser afectado emocionalmente, para poder enfrentar lo que Nordstrom y Robben (1995) llaman "choque existencial", definido como una desorientación sobre los límites entre la vida y la muerte específica de cada contexto en el que se vive, pues "escuchar, sentir, vivenciar el dolor del otro, o incluso sentir la imposibilidad de comprenderlo y dimensionarlo, hacen parte de los efectos que tiene el trabajo de campo en los investigadores" (De la O y Medina, 2012:18).

Existen varias consideraciones a tomar en cuenta, la primera es aprender a convivir con las emociones de nuestros sujetos y cultivar la calma durante las entrevistas que realicemos, pues de acuerdo a los investigadores entrevistados habrá ocasiones en las que tendremos que participar de charlas prolongadas sin que el informante brinde la información específica que estamos buscando, teniendo que lidiar con las emociones de los mismos cuando se tocan temas frágiles. En esos momentos debemos tratar de ser objetivos ante dichas emociones, pues esta situación puede moldear el análisis y el entendimiento de las investigaciones realizadas (Lee-Treweek y Linkogle, 2000).

Mientras se hace trabajo de gabinete $y$ antes de comenzar a envolverse dentro de la comunidad de estudio, es difícil dilucidar las cargas emocionales a las que uno se va a 
enfrentar, por eso es fundamental tener cuidado cuando se pide a las víctimas que narren su historia, pues habrá situaciones e historias de alto impacto para las que seguramente no estaremos preparados y es donde la estabilidad emocional del investigador se puede ver afectada debido al ambiente de estrés y angustia en el que se trabaja:

"Mis primeras entrevistas fueron muy difíciles, cuando empezaba a hacer las entrevistas y ver como se les empezaba a quebrar la voz, cómo empezaban a llorar, cómo rasgaban los sillones, cómo tomaban el café o todos esos detalles, regresaba a mi casa llorando. Ahí fue cuando decidí que tenía que hacer algo también por mi porque yo quería seguir con el tema sin embargo ya estaba siendo bastante afectada" (Gabriela. Comunicación personal, 22 de Agosto de 2016).

Ante estas situaciones, diversos investigadores narraron que entre las consecuencias que han tenido debido al ambiente emocional en el que han desarrollado sus investigaciones están las pesadillas, ansiedad, enfermedades, dolor de estómago, de espalda, insomnio y migrañas, por eso es importante buscar espacios de desahogo con compañeros, familiares o colegas y, si la situación lo amerita, es importante acudir a terapias psicológicas que ayuden a suavizar las impresiones que los contextos de violencia dejan como estragos. Daniela, quien trabajó con poblaciones callejeras optó por ver en su herramienta fundamental como antropóloga, el diario de campo, un lugar en el cual hacer catarsis y plasmar por escrito todo lo que sentía.

Por último, la reflexión personal sobre ¿Cuál es mi objeto de estudio?, ¿Por qué estoy aquí?, pero sobre todo ¿Cuál es mi papel como investigador? es fundamental hacerse estas preguntas para no caer en el peligroso juego de prometer que se va a cambiar la realidad que se estudia y crear falsas esperanzas con la comunidad. Sobre esto, Daniela menciona la dificultad de darse cuenta de su papel como investigadora, pues tuvo que callar y no denunciar delitos como pederastia $\mathrm{y}$ otros abusos con el fin de no crear más problemas de los que ya había en su campo de estudio.

Hacernos las preguntas anteriores tiene entonces la intensión de analizar cuáles son las motivaciones que impulsan la investigación y cuáles son los alcances de la misma. La antropología comprometida, menciona Falla (en Nanz 1995), implica dar algo de regreso a la gente, de esta forma, el reto principal es buscar que nuestras investigaciones como científicos sociales generen algún impacto positivo para disminuir la violencia, pues de nada serviría 
escribir si esas investigaciones nunca saldrán de la universidad y sólo serán leídas por otros académicos.

"Idealmente, el investigador debe ofrecer una oportunidad a la gente de ver cómo su voz es reflejada en su trabajo. Muchas cosas podrían parecer obtusas para ellos, sin embargo, el trabajo aún puede ser muy útil para otra audiencia que pueda retribuir a la comunidad [... $\mathrm{La}$ investigación en zonas de conflicto acerca al antropólogo a la comunidad. Es complementar la antropología con un cierto sentido de justicia" (Falla en Nanz, 1995:273).

\section{APUNTES DE CIERRE}

La situación que enfrenta el país desde que se declaró la Guerra contra el Narcotráfico, representa un desafío para el trabajo de investigación, pues este contexto ha redefinido las condiciones de vida y las formas de hacer investigación. Frente a este escenario, Sandoval (2011) plantea:

¿No será que en el marco de la realidad de violencia, inseguridad e impunidad generalizada que vive el país, la antropología tiene la gran oportunidad de hacer aportaciones más explícitas a partir de sus características más básicas?” (Sandoval, 2011:59).
Para los científicos sociales es necesario comprender la dimensión y los grandes retos que representa el contexto actual del país para nuestro quehacer.

Nuestro entrenamiento como etnógrafos debe permitirnos usar nuestras herramientas epistémicas aprendidas para la recolección de datos en caso de cualquier ataque, pues nuestro adiestramiento en la descripción densa de paisajes, personas y situaciones podría en algún momento salvarnos y ayudar a dar señas específicas en caso de sufrir alguna agresión. Al respecto, Winkler y Hanke (1995) señalan que sus antecedentes como antropóloga le ayudaron a recolectar datos en contra de su violador durante trabajo de campo.

$$
\text { Por otro lado, y ante las }
$$
adversidades que se nos presentan en estos tiempos, surge la necesidad de generar una red académica de estudiantes y profesores que estén haciendo trabajo de campo. Esto con la intención de establecer una relación que nos permita enterarnos de lo que otros están haciendo, cómo y dónde, para así poder monitorear y apoyarnos colectivamente. Del mismo modo, existe también la urgencia de preparar a los antropólogos en formación para superar el miedo y la frustración que implica tratar de hacer trabajo de campo en situaciones de riesgo, pues, como se ha revisado, la investigación en zonas de conflicto implica, 
además de una serie de precauciones, conocer las posibles limitantes con las que nos podemos enfrentar. Aceptar que siempre habrá huecos que no podrán ser llenados, y estar abiertos, por un lado, a armar el rompecabezas lo mejor que se pueda con la poca información que se obtiene bajo estos escenarios, y por el otro, a tomar en cada momento la dirección que presente menos resistencia, pues habrá ocasiones en las que tendremos que redefinir prioridades y elegir entre la información que deseamos obtener y la propia seguridad.

Por último, debemos tomar en cuenta que el mundo no se está convirtiendo en un lugar más seguro y que el trabajo de campo sí es posible incluso en el contexto más peligroso (Feldman, 1995). Es por eso que, desde la característica más básica de la disciplina, debemos asumir un compromiso ético, haciendo lo que Rosemberg (2012) llama "etnografías de indignación", encontrar en ellas un lugar de militancia, recuperar las voces de las víctimas y usar las monografías como sitios de resistencia en las que se haga un llamado a la justicia y en contra del terror.

\section{Referencias}

COMISIÓN INTERAMERICANA

DE DERECHOS HUMANOS.

Situación de los Derechos Humanos en México, 2015.

DURAND, Jorge. "Coordenadas metodológicas. De cómo armar el rompecabezas". En: OEHMICHEN, Christina (Ed.), Etnografía y trabajo de campo en las Ciencias Sociales, México: Universidad Nacional Autónoma de México, Instituto de Investigaciones Antropológicas, 2014, Pp. 261-284, 2014.

DURÍN, Severin. "Los que la guerra desplazó: Familias del noreste de México en el exilio". Desacatos, núm. 38, Pp. 29-42, 2012.

DE LA O, María Eugenia y MEDINA, Nora. "Ser joven en la frontera norte de México".

Desacatos, núm. 38, Pp. 181-190, 2012.

EMERSON, Robert, FRETZ, Rachel y SHAW, Linda. Writing ethnographic Fieldnotes. $2^{\mathrm{a}} \mathrm{Ed}$. Chicago, University of Chicago Press, 2011.

FEIXA, Carles y FERRÁNDIZ, Francisco. "Una mirada antropológica sobre las violencias". 
Alteridades. v. 14, n. 027, Pp. 159174, 2004.

FELDMAN, Allen. "Ethnographic

States of Emergency". En

NORDSTROM, Carolyn y

ROBBEN, Antonius (Ed.), Fieldwork

Under Fire. Berkeley: University of

California Press, 1995, Pp. 224-253.

FERRÁNDIZ, Francisco. "La

etnografía como campo de minas: De

las violencias cotidianas a los

paisajes posbélicos". En: BULLEN,

Margaret y DIEZ, Carmen (Coords.),

Retos teóricos y Nuevas Prácticas,

col. XI Congreso de Antropología de

la FAAEE, Donostia, Ankulegi

Antropologia Elkartea, Pp. 89-116,

2008. [en línea]

<http://hedatuz.euskomedia.org/5332

/1/14089115.pdf>

GASKEW, Tony. "Are you with the FBI? Fieldwork challenges in a post 9/11 muslim-american community".

Fieldwork Under Difficult

Circumstances. Practicing

Anthropology, v. 31, n. 2, Pp.12-17, 2009.

GAVILÁN, Lurgio. Memorias de un soldado desconocido. Autobiografía y antropología de la violencia.
México, Universidad Iberoamericana, 2012.

GILL, Hannah. "Finding a Middle

Ground Between Extremes: Notes on

Researching Transnational Crime and

Violence", Antrhopology Matters

Journal, v. 6, n. 2, Pp.1-9, 2004.

GILLES, Bataillon. "Narcotráfico y corrupción: Las formas de la violencia en México en el siglo XXI". Nueva Sociedad, n. 255, Pp. 54-68, 2015.

GOLDSTEIN, Daniel. Qualitative Research in Dangerous Places:

Becoming an Ethnographer of

Violence and Personal Dafety, Social

Science Research Council, 2014.

GREEN, Linda. "Living in a State of Fear". En NORDSTROM, Carolyn y ROBBEN, Antonius (Ed.) Fieldwork Under Fire. Berkely: University of California Press, 1995, Pp. 105-128.

HOWELL, Nancy. "Human Hazards of Fieldwork". En ROBBEN, Antonius y SLUKA Jeffrey (Ed.)

Ethnographic Fieldwork: An

Anthropological Reader. Malden:

Wiley-Blackwell, 2012, Pp. 234-244.

HAMMERSLEY, Martyn y

ATKINSON, Paul. Etnografía. 
Métodos de investigación, Barcelona, Paidós, 1994.

JIMENO, Myriam, VARELA,

Daniel, CASTILLO, Ángela.

"Experiencias de violencia:

etnografía y recomposición social en

Colombia”. Sociedade e Cultura. V.

14, n. 2, Pp. 275-285, 2011.

KOVATS-BERNAT, Christopher.

"Negotiating Dangerous Fields:

Pragmatic Strategies for Fieldwork amid Violence and Terror".

American Anthropologist, Vol. 104,

No. 1, 2002.

LEE-TREWEEK, Geraldine y

LINKOGLE, Stephanie. "Putting

danger in the Frame". En: LEE-

TREWEEK, Geraldine y

LINKOGLE, Stephanie (Ed.).

Danger in the Field. Risk and ethics

in social research, New York:

Routledge, 2000, Pp. 8-25.

MANZ, Beatriz, "Reflections on an

Antropología Comprometida:

Conversations with Ricardo Falla".

En NORDSTROM, Carolyn y

ROBBEN, Antonius. Fieldwork

under Fire. Berkeley: University of

California Press, 1995, Pp. 261-275.
MARTÍNEZ, Juan. Ver, oír, callar:

Un año con la Mara Salvatrucha 13.

La Rioja, Pepitas de Calabaza, 2015.

NASH, June. "Ethnology in a

Revolutionary Setting” En ROBBEN, Antonius y SLUKA, Jeffrey (Ed.),

Ethnographic Fieldwork: An

Anthropological Reader, Malden:

Wiley-Blackwell, 2012, Pp. 244-255.

NATERAS, Alfredo. "Etnografías de violencia y muerte: La Mara

Salvatrucha y el Barrio 18.

Iztapalapa, Revista de Ciencias y

Humanidades. n.69. Pp. 87-108,

2010.

NORDSTROM, Carolyn. "War on

the Front Lines". En:

NORDSTROM, Carolyn y

ROBBEN, Antonius (Eds.),

Fieldwork Under Fire, Berkeley:

University of California Press, 1995,

Pp. 129-154.

NORDSTROM, Carolyn y

ROBBEN, Antonius. Fieldwork

under Fire. Berkeley: University of

California Press, 1995.

PEREYRA, Guillermo. "México:

violencia criminal y Guerra contra el

Narcotráfico". Revista Mexicana de 
Sociología, v.74. n. 3, Pp.429-460, 2012.

PÉREZ, Jorge Enrique. “La guerra contra el narcotráfico: ¿una guerra perdida?”. Espacios Públicos. V.14, n. 30, Pp. 211-230, 2011.

OLUJIC, Maria. "The Croatian War Experience". En NORDSTROM, Carolyn y ROBBEN, Antonius (Eds.), Fieldwork Under Fire. Berkeley: University of California Press, 1995, Pp. 186-205.

OMIDIAN, Patricia. "Living and Working in a Warzone: An applied anthropologist in Afghanistan.

Fieldwork Under Difficult

Circumstances". Practicing

Anthropology. V. 31, n. 2, Pp. 4-11, 2009.

PIEKE, Frank. "Witnessing the 1989

Chinese people's movement". En

NORDSTROM Carolyn y ROBBEN, Antonius (Eds.), Fieldwork under Fire, Berkeley: University of California Press, 1995, Pp. 62-80.

RODGERS, Dennis. "Haciendo del

peligro una vocación: la antropología, la violencia y los dilemas de la observación participante". Revista Española de
Investigación Criminológica, v. 2, n.1, Pp. 1-24, 1997.

ROSEMBERG, Florence.

Conferencia Magistral "La etnografía en tiempos de violencia". La etnografía y los desafíos del México contemporáneo, INAH TV, 2012. [https://www.youtube.com/watch?v= UqqhGb4-ujU\&t=3823s]

SANDOVAL, Efrén. "Economía de la fayuca y del narcotráfico en el noreste de México. Extorsiones, contubernios y solidaridades en las economías transfronterizas".

Desacatos, núm. 38, Pp. 43-60, 2012.

SWEDENBURG, Ted. "With Genet in the Palestitian Field". En NORDSTROM, Carolyn y ROBBEN, Antonius (Eds.), Fieldwork Under Fire, Berkeley: University of California Press, Pp.2543, 1995.

VALENZUELA, José Manuel.

"Narcocultura, violencia y ciencias socioantropológicas". Desacatos, n. 38, Pp. 95-102, 2012.

WINKLER, Cathy y HANKE, Penelope. "Ethnography of Ethnographer". En NORDSTROM, Carolyn, ROBBEN, Antonius, 
Fieldwork under Fire, Berkeley:

University of California Press, Pp.

155-185, 1995.

WOLF, Sonja. "La guerra de México

contra el narcotráfico y la Iniciativa

Mérida: piedras angulares en la

búsqueda de legitimidad". Foro

Internacional, v. 51, n. 4, Pp. 669-

$714,2011$.

ZAVALA, Aurora. "Mi llegada al

paraíso. Una etnografía entre

pandillas". En: OEHMICHEN,

Cristina, La etnografía y el trabajo de

campo en las ciencias sociales,

México: Universidad Nacional

Autónoma de México, Instituto de

Investigaciones Antropológicas,

2014, Pp. 241-260. 